\title{
Exotic Low-Energy Excitations Emergent in the Random Kitaev Magnet $\mathrm{Cu}_{2} \mathrm{IrO}_{3}$
}

\author{
Y. S. Choi, ${ }^{1}$ C. H. Lee, ${ }^{1}$ S. Lee, ${ }^{1}$ Sungwon Yoon, ${ }^{1}$ W.-J. Lee, ${ }^{1}$ J. Park, ${ }^{1}$ Anzar Ali, ${ }^{2}$ Yogesh Singh, ${ }^{2}$ \\ Jean-Christophe Orain, ${ }^{3}$ Gareoung Kim, ${ }^{4}$ Jong-Soo Rhyee, ${ }^{4}$ Wei-Tin Chen, ${ }^{5}$ \\ Fangcheng Chou, ${ }^{5,6,7}$ and Kwang-Yong Choi ${ }^{1, *}$ \\ ${ }^{1}$ Department of Physics, Chung-Ang University, Seoul 06974, Republic of Korea \\ ${ }^{2}$ Department of Physical Sciences, Indian Institute of Science Education and Research Mohali, \\ Sector 81, S. A. S. Nagar, Manauli 140306, India \\ ${ }^{3}$ Laboratory for Muon Spin Spectroscopy, Paul Scherrer Institute, 5232 Villigen PSI, Switzerland \\ ${ }^{4}$ Department of Applied Physics, Kyung Hee University, Yongin 17104, Republic of Korea \\ ${ }^{5}$ Center for Condensed Matter Sciences, National Taiwan University, Taipei 10617, Taiwan \\ ${ }^{6}$ National Synchrotron Radiation Research Center, Hsinchu 30076, Taiwan \\ ${ }^{7}$ Taiwan Consortium of Emergent Crystalline Materials, Ministry of Science and Technology, Taipei 10622, Taiwan
}

(Received 15 November 2018; revised manuscript received 17 February 2019; published 26 April 2019)

\begin{abstract}
We report on magnetization $M(H)$, dc and ac magnetic susceptibility $\chi(T)$, specific heat $C_{m}(T)$ and muon spin relaxation $(\mu \mathrm{SR})$ measurements of the Kitaev honeycomb iridate $\mathrm{Cu}_{2} \mathrm{IrO}_{3}$ with quenched disorder. In spite of the chemical disorders, we find no indication of spin glass down to $260 \mathrm{mK}$ from the $C_{m}(T)$ and $\mu \mathrm{SR}$ data. Furthermore, a persistent spin dynamics observed by the zero-field muon spin relaxation evidences an absence of static magnetism. The remarkable observation is a scaling relation of $\chi[H, T]$ and $M[H, T]$ in $H / T$ with the scaling exponent $\alpha=0.26-0.28$, expected from bond randomness. However, $C_{m}[H, T] / T$ disobeys the predicted universal scaling law, pointing towards the presence of additional low-lying excitations on the background of bond-disordered spin liquid. Our results signify a many-faceted impact of quenched disorder in a Kitaev spin system due to its peculiar bond character.
\end{abstract}

DOI: $10.1103 /$ PhysRevLett.122.167202

The exactly solvable Kitaev honeycomb model provides a novel route to achieve elusive topological and quantum spin liquids [1,2]. Exchange frustration of bond-dependent Ising interactions fractionalizes the $j_{\text {eff }}=\frac{1}{2}$ spin into itinerant Majorana fermion and static $Z_{2}$ gauge flux [3-5]. Edge sharing of octahedrally coordinated metal ions subject to strong spin-orbit coupling supports the realization of Kitaev-type interactions [6-8].

In the quest for a Kitaev honeycomb magnet, the family of $A_{2} \mathrm{IrO}_{3}(A=\mathrm{Na}, \mathrm{Li})$ and $\alpha-\mathrm{RuCl}_{3}$ are considered prime candidate materials [9-16]. In these compounds, however, the theoretically predicted spin-liquid state is preempted by long-range magnetic order due to structural imperfections. As the real materials are vulnerable to a monoclinic stacking of honeycomb layers, non-Kitaev terms inevitably occur. A related issue is to engineer local crystal environments towards an optimal geometry to minimize the nonKitaev interactions.

Very recently, the new Kitaev honeycomb iridates $\mathrm{H}_{3} \mathrm{LiIr}_{2} \mathrm{O}_{6}$ and $\mathrm{Cu}_{2} \mathrm{IrO}_{3}$ have been derived from their ancestors $\mathrm{A}_{2} \mathrm{IrO}_{3}$ through soft structural modifications $[17,18] . \mathrm{H}_{3} \mathrm{LiIr}_{2} \mathrm{O}_{6}$ is obtained by replacing the interlayer $\mathrm{Li}^{+}$ions with $\mathrm{H}^{+}$from $\alpha-\mathrm{Li}_{2} \mathrm{IrO}_{3}$, while the honeycomb layer remains intact. A scaling of the specific heat and NMR relaxation rate gives evidence for the presence of fermionic excitations [17]. In the H-intercalated iridate, hydrogen disorders bring about magnetic bond disorders and suppress longer-range exchange interactions, thereby disrupting the long-range magnetic order $[19,20]$. In the case of $\mathrm{Cu}_{2} \mathrm{IrO}_{3}$, all of the $A$-site cations of $\mathrm{Na}_{2} \mathrm{IrO}_{3}$ are permuted by the $\mathrm{Cu}^{+}$ions, which are located at the center of the hexagonal plaquette as well as between the honeycomb layers.

Figure 1(a) presents the crystal structure of $\mathrm{Cu}_{2} \mathrm{IrO}_{3}(\mathrm{C} 2 /$ $c$ space group), in which the honeycomb layers are stacked by $\mathrm{CuO}_{2}$ dumbbells, distinct from $\mathrm{CuO}_{6}$ octahedra within the honeycomb layers. The interlayer dumbbell structure arises from the eclipsed stacking of adjacent layers that align the oxygen and interlayer copper atoms in a line. We note that the $\mathrm{CuO}_{2}$ dumbbells in $\mathrm{Cu}_{2} \mathrm{IrO}_{3}$ and the linear $\mathrm{O}-\mathrm{H}-\mathrm{O}$ links in $\mathrm{H}_{3} \mathrm{LiIr}_{2} \mathrm{O}_{6}$ have structural similarities in bridging the stacking of honeycomb layers. This structural alteration leads to an elongation of the $c$ axis and closeness of the Ir-Ir-Ir bond angles to the ideal $120^{\circ}$, compared to its predecessor $\mathrm{Na}_{2} \mathrm{IrO}_{3}$ [see Fig. 1(b)]. However, little is known about its underlying magnetism.

In this Letter, we provide thermodynamic and $\mu \mathrm{SR}$ spectroscopic signatures of a bond disordered spin-liquid state. We find a scaling relation of the $(T, H)$-dependent magnetization and ac susceptibility in $T / H$, but not of the 


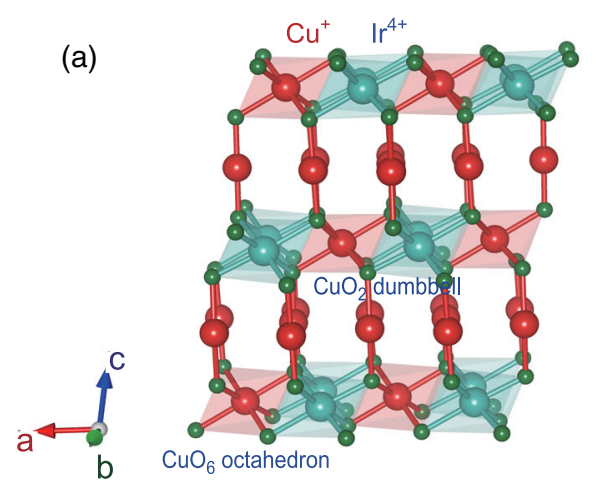

(c)

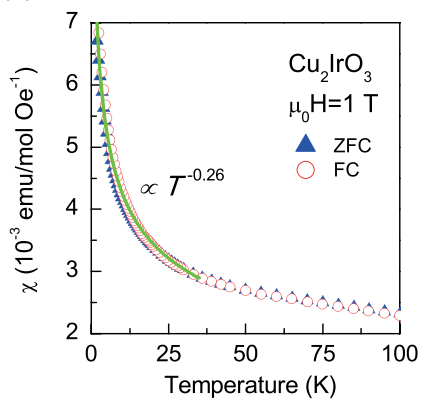

(d)

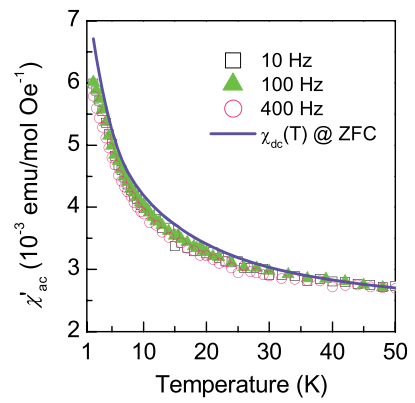

(b)

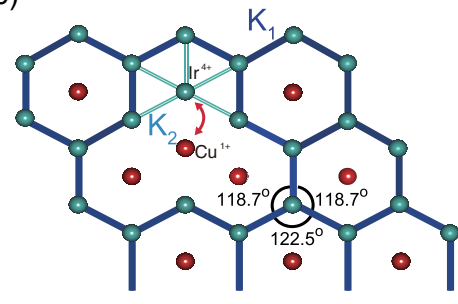

$\mathrm{Cu}^{+} / / \mathrm{r}^{4+}$ site disorder

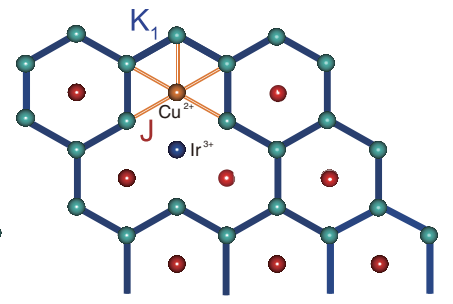

$\mathrm{Cu}^{+} / \mathrm{Cu}^{2+}$ mixed valence (e)

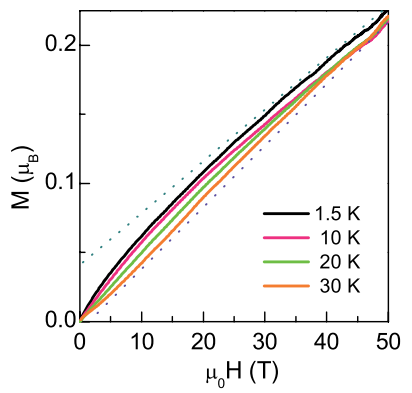

(f)

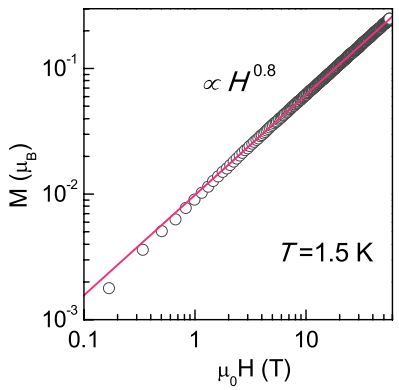

FIG. 1. (a) Crystal structure of $\mathrm{Cu}_{2} \mathrm{IrO}_{3}$ consisting of edge-sharing $\left(\mathrm{Ir}_{2 / 3} \mathrm{Cu}_{1 / 3}\right) \mathrm{O}_{6}$ octahedra in the honeycomb layers and linear $\mathrm{CuO} 2$ dumbbells between the layers. The greenish blue, red, and green spheres represent $\mathrm{Ir}, \mathrm{Cu}$, and $\mathrm{O}$ ions, respectively. (b) Sketch of two types of chemical disorders occurring in the honeycomb plane. $\mathrm{Cu}^{1+} / \mathrm{Ir}^{4+}$ cation disorders and $\mathrm{Cu}^{1+}\left(\mathrm{Ir}^{4+}\right) / \mathrm{Cu}^{2+}\left(\mathrm{Ir}^{3+}\right)$ mixed valence not only create nonmagnetic impurities but also supply a new triangular motif. (c) $T$ dependence of the static magnetic susceptibility of $\mathrm{Cu}_{2} \mathrm{IrO}_{3}$ measured at $\mu_{0} H=1 \mathrm{~T}$ in zero-field-cooled (ZFC) and field-cooled processes. The green solid line is a fit to a power-law $\chi(T) \sim T^{-\alpha_{s}}$ with $\alpha_{s} \approx 0.26$. (d) $T$ dependence of the ac magnetic susceptibility at selected frequencies $\nu=10,100$, and $400 \mathrm{~Hz}$ and with oscillating field $H_{\mathrm{osc}}=10$ Oe plotted together with the ZFC dc magnetic susceptibility. (e) High-field magnetization curves $M(H)$ measured at $T=1.5,10,20$, and $30 \mathrm{~K}$. (f) Log-log plot of $M(H)$ at $T=1.5 \mathrm{~K}$. The solid red line is a power-law fit $M(H) \sim H^{1-\alpha_{m}}$ with $\alpha_{m} \approx 0.2$.

$(T, H)$-dependent specific heat. This observation demonstrates a many-faceted role of bond randomness in the Kitaev quantum magnet.

Polycrystalline samples of $\mathrm{Cu}_{2} \mathrm{IrO}_{3}$ were prepared by a topotactic reaction as described in Ref. [18]. dc and ac magnetic susceptibilities were measured using a SQUID and a vibrating sample magnetometer (Quantum Design MPMS and VSM). The magnetization measurements up to 14 Twere carried out using a Physical Property Measurement System (Quantum Design PPMS Dynacool). Specific heat was measured with the thermal-relaxation method using an option of the PPMS apparatus with a ${ }^{3} \mathrm{He}$ insert. High-field magnetization curves were recorded with a nondestructive pulsed magnet at the Dresden High Magnetic Field Laboratory. Zero-field (ZF)- and longitudinal-field (LF)$\mu \mathrm{SR}$ experiments were performed with the DOLLY spectrometer at PSI (Villigen, Switzerland). For measurements down to $0.26 \mathrm{~K}$, the samples were loaded into the Variox cryostat equipped with Heliox insert. All of the obtained $\mu \mathrm{SR}$ data were analyzed using the software package MusRfit [21].

Figure 1(c) presents the $T$ dependence of the static magnetic susceptibility $\chi(T)$ of $\mathrm{Cu}_{2} \mathrm{IrO}_{3}$ measured at $\mu_{0} H=1 \mathrm{~T}$ in zero-field-cooled (ZFC) and field-cooled (FC) processes. The $\mathrm{ZFC}$ and $\mathrm{FC} \chi(T)$ data show no apparent difference. As $T \rightarrow 0 \mathrm{~K}, \chi(T)$ exhibits a steep increase without obvious saturation or kink, in spite of the large Curie-Weiss temperature $\Theta_{\mathrm{CW}}=-110 \mathrm{~K}$ [18]. It is remarkable that $\chi(T)$ obeys a power-law increase $\chi(T) \sim$ $T^{-\alpha_{s}}\left(\alpha_{s} \approx 0.26\right)$ with no hint of the Curie tail for temperatures below $30 \mathrm{~K}$. Such a sub-Curie law behavior points towards the presence of abundant low-energy excitations. Shown in Fig. 1(d) is the $T$ dependence of the real component of ac susceptibility $\chi_{\text {ac }}^{\prime}(T)$ at selected frequencies $\nu=10,100$, and $400 \mathrm{~Hz}$ with no external dc field. $\chi_{\text {ac }}^{\prime}(T)$ displays neither sharp peak nor discernible frequency dependence. Obviously, this together with no bifurcation between the ZFC and FC $\chi(T)$ rules out the occurrence of slow dynamics such as a spin frozen or glasslike state. As seen in Fig. 1(e), the high-field magnetization curves $M(H)$ at selected low temperatures clearly deviate from a linear- $H$ dependence. Significantly, the $T=1.5 \mathrm{~K} M(H)$ follows a power-law dependence $M(H) \sim H^{1-\alpha_{m}}$ with $\alpha_{m} \approx 0.2$ over an entirely measured field range [see a log-log plot of $M(H)$ in Fig. 1(f)]. The commonly observed power-law behavior of $\chi(T)$ and $M(H)$ with $\alpha_{s} \approx \alpha_{m}$ constitutes a hallmark of 
random singlet, bond-disordered spin liquid, and valence bond with quenched disorders [22-26].

In the case of $\mathrm{Cu}_{2} \mathrm{IrO}_{3}$, quenched disorders are mainly caused by the distinct coordination environment of the $\mathrm{Cu}$ ions. As shown in Fig. 1(a), the $\mathrm{Cu}$ atoms within (between) the honeycomb layers are in octahedral (dumbbell) coordination. In contrast to the dumbbell coordination, an octahedral environment can accommodate both $\mathrm{Cu}^{+}$and $\mathrm{Cu}^{2+}$ valence states [18]. Consequently, there appear inplane chemical disorders: magnetic ions (either $\mathrm{Ir}^{4+}$ or $\mathrm{Cu}^{2+}$ ) occupy randomly at the center of the honeycomb lattice, while nonmagnetic ions (either $\mathrm{Cu}^{1+}$ or $\mathrm{Ir}^{3+}$ ) go on the vertex of the honeycomb lattice. Supportive evidence for the intralayer quenched disorders comes from the power-law dependence of both $\chi(T)$ and $M(H)$ persisting down to at least $2 \mathrm{~K}$ and $0.3 \mathrm{~T}$, respectively. We stress that the low- $T \chi(T)$ and the low- $H M(H)$ contain no Curie tail and Brillouin-function-like increase, respectively, which may arise from the interlayer orphan spins that are not exchange coupled. As such, as sketched in Fig. 1(b), an impact of the quenched disorders on magnetism is twofold. One is to introduce spin vacancies, and the other is to generate a spin triangular motif which has an exchange interaction different from the original honeycomb lattice, thereby creating a disparate randomness in exchange coupling constants.

Generically, quenched disorder can generate a spin glass state or a bond-disordered quantum spin liquid, which may be static or dynamic in nature $[22,23]$. To differentiate between these possibilities, we performed $\mu \mathrm{SR}$ measurements.

Figures 2(a) and 2(b) show the ZF- $\mu \mathrm{SR}$ spectra at selected temperatures and the LF- $\mu \mathrm{SR}$ spectra measured at $T=$ $0.26 \mathrm{~K}$ in an applied field of $H=0-4500 \mathrm{G}$. The $\mu \mathrm{SR}$ data are successfully modeled with the relaxation function $P_{\mathrm{ZF}}(t)=G_{\mathrm{KT}}(t) \exp \left[-\left(\lambda_{\mathrm{ZF}} t\right)^{\beta}\right]+P_{\mathrm{bg}}$, where $G_{\mathrm{KT}}(t)=\frac{1}{3}+$ $\frac{2}{3}\left(1-\Delta^{2} t^{2}\right) \exp \left(-\frac{1}{2} \Delta^{2} t^{2}\right)$ is the Gaussian Kubo-Toyabe function. Fitting of the ZF data yields $\Delta \approx 0.12 \mathrm{MHz}$, typical for depolarization by nuclear moments [27]. Here, $\lambda_{\mathrm{ZF}}$ represents the muon relaxation rate caused by the electronic Ir spins. The $T$-independent $P_{\mathrm{bg}}$ term captures a background contribution, which originates from muons implanted in the silver sample holder or the cryostat.

With decreasing temperature, the ZF- $\mu$ SR spectra gradually change their asymmetry line shape from a Gaussian- to a Lorentzian-like relaxation form. At the measured lowest temperature of $0.26 \mathrm{~K}$, we observe no signatures of static magnetism. More specifically, this spectrum contains no oscillatory component, no missing asymmetry [see an enlarged view in the inset of Fig 2(a)], and there is no indication to a recovery of a $1 / 3$ tail at long times. Furthermore, the LF dependence of the $\mu$ SR spectra ascertains the notion that the Ir moments remain in a dynamically fluctuating state at least down to $T=0.26 \mathrm{~K}$. As shown in Fig. 2(b), the $H_{\mathrm{LF}}=4500 \mathrm{G}$ spectrum still shows significant relaxation. This result is in sharp contrast to expectations for a
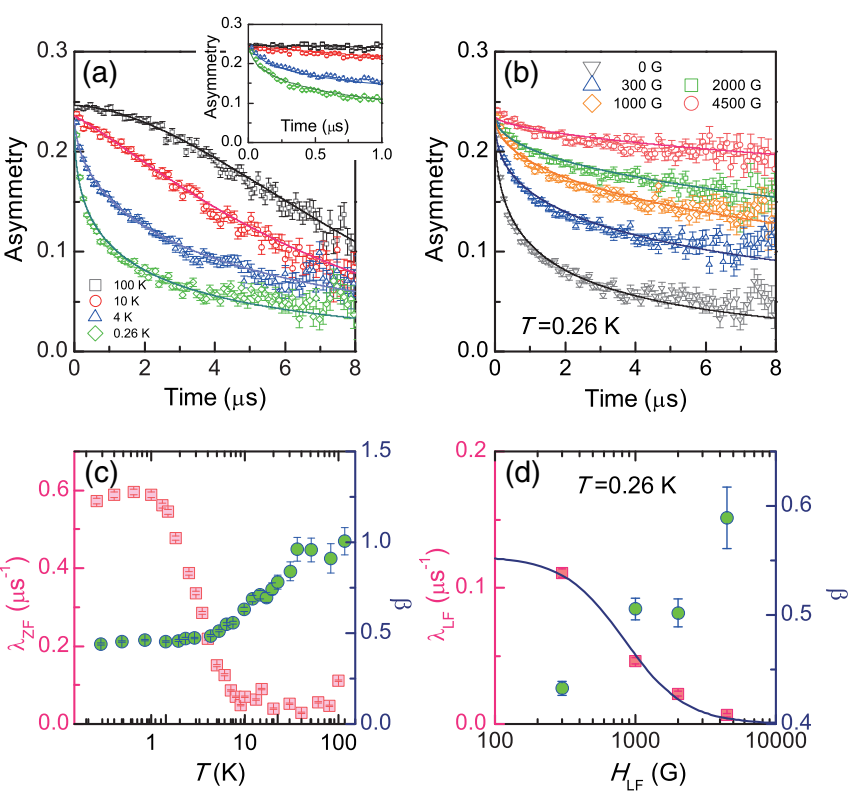

FIG. 2. (a) $\mathrm{ZF}-\mu \mathrm{SR}$ spectra of $\mathrm{Cu}_{2} \mathrm{IrO}_{3}$ at selected temperatures. The inset is an enlargement of the early-time spectra. (b) Field dependence of LF- $\mu \mathrm{SR}$ spectra measured at $T=0.26 \mathrm{~K}$ in an applied field of $H=0-4500 \mathrm{G}$. The solid lines are fits to the relaxation function described in the text. (c) The muon relaxation rate $\lambda_{\mathrm{ZF}}(T)$ and the stretch exponent $\beta(T)$ extracted from fitting to the ZF data. (d) Longitudinal-field dependence of the muon relaxation rate $\lambda_{\mathrm{LF}}\left(H_{\mathrm{LF}}\right)$ and the stretch exponent $\beta\left(H_{\mathrm{LF}}\right)$ extracted from the $T=0.26 \mathrm{~K} \mathrm{LF}$ data. The solid curve denotes the fit of $\lambda_{\mathrm{LF}}\left(H_{\mathrm{LF}}\right)$ to the Redfield formula.

static ZF relaxation mechanism. From the saturated low- $T$ $\lambda_{\mathrm{ZF}} \approx 0.6 \mathrm{MHz}$ [see Fig. 2(c)], we estimate the local field $B_{\mathrm{loc}}=\lambda_{\mathrm{ZF}} / \gamma_{\mu} \approx 7.04 \mathrm{G}$ with the muon gyromagnetic ratio $\gamma_{\mu} / 2 \pi=135.5 \mathrm{MHz} / \mathrm{T}$ and, therefore, full muon polarization is expected when $H_{\mathrm{LF}} \geq 70 \mathrm{G}$ [27].

We next turn to the $T$ and $H$ evolution of the obtained fit parameters. As seen from Fig. 2(c), with decreasing temperature $\lambda_{\mathrm{ZF}}(T)$ starts to increase at $6 \mathrm{~K}$ and then saturates at about $1 \mathrm{~K}$. Such a low- $T$ plateau in $\lambda_{\mathrm{ZF}}(T)$, representing a persistent spin dynamics, has been observed in a range of geometrically frustrated magnets exhibiting spin freezing, weak magnetic order, or even quantum spin liquid behavior [28-31].

In parallel to $\lambda_{\mathrm{ZF}}(T)$, the stretching exponent decreases rapidly from $\beta \approx 1$ on cooling through $50 \mathrm{~K}$, and then levels off to $\beta \approx 0.5$ below $6 \mathrm{~K}$. The obtained value of $\beta$ at the base temperature is larger than the $\beta=1 / 3$ (value of canonical spin glass) [32].

Shown in Fig. 2(d) is the LF dependence of $\lambda_{\mathrm{LF}}\left(H_{\mathrm{LF}}\right)$ and $\beta\left(H_{\mathrm{LF}}\right)$ measured at $T=0.26 \mathrm{~K}$. In the Redfield model, $\lambda_{\mathrm{LF}}(T)$ is associated with the fluctuation frequency $\nu$ and the fluctuating time-averaged local field $\left\langle H_{\text {loc }}^{2}\right\rangle$ by $\lambda_{\mathrm{LF}}\left(H_{\mathrm{LF}}\right)=2 \gamma_{\mu}^{2}\left\langle H_{\mathrm{loc}}^{2}\right\rangle \nu /\left(\nu^{2}+\gamma_{\mu}^{2} H_{\mathrm{LF}}^{2}\right)$ [27,33]. From fits of the $T=0.26 \mathrm{~K}$ data, we obtain $\nu \sim 72 \mathrm{MHz}$ and $H_{\text {loc }} \sim 25 \mathrm{G}$. The determined $\nu\left(H_{\mathrm{loc}}\right)$ is roughly two times slower (larger) than $\nu \sim 150 \mathrm{MHz}$ and $H_{\mathrm{loc}} \sim 18 \mathrm{G}$ of the 
well-investigated spin-liquid material $\mathrm{ZnCu}_{3}(\mathrm{OH})_{6} \mathrm{Cl}_{2}$ [29]. On the LF application, $\beta\left(H_{\mathrm{LF}}\right)$ steadily increases, implying the gradual quenching of widely distributed fluctuating local fields. Taken the $\mu \mathrm{SR}$ data together, we conclude that $\mathrm{Cu}_{2} \mathrm{IrO}_{3}$ is a close realization of a bond-disordered spin liquid.

In the following, we will discuss an intricate relation between quenched disorder and critical spin correlations.

In the inset of Fig. 3(a), the $H$ and $T$ dependences of $\chi_{\mathrm{ac}}^{\prime}$ measured at $\nu=100 \mathrm{~Hz}$ are plotted on the log-log scale in the applied fields of $\mu_{0} H=0-3 \mathrm{~T}$. With increasing field, $\chi_{\mathrm{ac}}^{\prime}(T)$ is systematically reduced, yet still maintains a power-law dependence, while changing the exponent from $\alpha_{s}=0.26$ at $0 \mathrm{~T}$ to 0.14 to $3 \mathrm{~T}$. In an attempt to corroborate the dynamic scaling behavior, we plot $T^{\alpha_{s}} \chi_{\mathrm{ac}}^{\prime}$ vs $H / T$ in Fig. 3(a). Strikingly, the $(H, T)$-dependent $\chi_{\text {ac }}^{\prime}$ data overlap over 3 orders of magnitude with the same value of $\alpha_{s}=0.26$. We provide further supportive evidence for universal scaling behavior by testing the similar scaling behavior in the $M(H)$ data measured up to $\mu_{0} H=14 \mathrm{~T}$ at various temperatures $(2,5,10,20,30$, and $47 \mathrm{~K})$. We plot $M T^{\alpha_{m}-1}$ against $H / T$ in Fig. 3(b), and obtain the exponent value of $\alpha_{m}=0.28$ that guarantees a sufficient data collapse onto a single scaling curve. The scaling exponent of $0.26-0.28$ agrees exceptionally well between the two distinct thermodynamic quantities. This is reminiscent of universal scaling observed in a certain class of frustrated
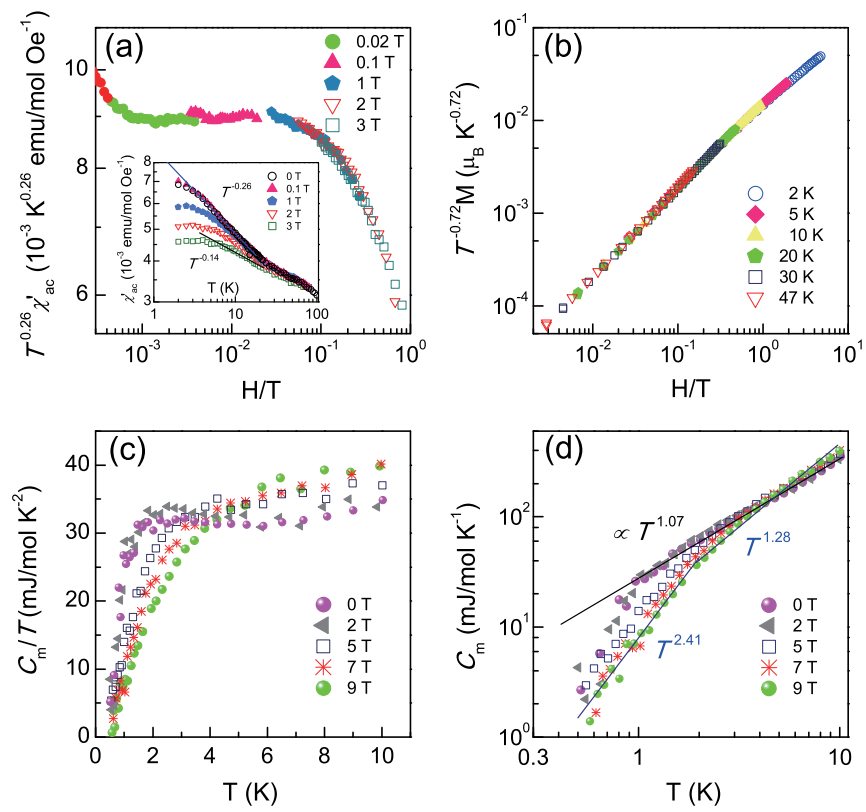

FIG. 3. (a) Log-log scaled plot of $H^{0.26} \chi_{\text {ac }}^{\prime}$ vs $T / H$. The inset plots the real component of the ac susceptibility $\chi_{\mathrm{ac}}^{\prime}(T)$ vs temperature under various external dc fields. The solid line is a power-law dependence of $\chi_{\mathrm{ac}}^{\prime}(T)$. (b) $T-H$ scaling of $M(H)$ plotted on a log-log scale. (c) Magnetic specific heat divided by temperature $C_{m} / T$ vs $T$ for various magnetic fields. (d) Log-log plot of the same $(T, H)$-dependent $C_{m}$. The solid lines are the fits of the $C_{m}$ data to a power law. quantum magnets $\mathrm{H}_{3} \mathrm{LiIr}_{2} \mathrm{O}_{6}, \quad \mathrm{LiZn}_{2} \mathrm{Mo}_{3} \mathrm{O}_{8}, \quad$ and $\mathrm{ZnCu}_{3}(\mathrm{OH})_{6} \mathrm{Cl}_{2}$, which commonly feature quantum spin liquids and quenched disorders [24].

We further explore the specific heat scaling. Figure 3(c) shows a $C_{m} / T$ vs $T$ plot at low temperatures of $T=0.5-10 \mathrm{~K}$ under the external magnetic fields of $\mu_{0} H=0-9 \mathrm{~T}$. The zero-field $C_{m} / T$ data show little variation with temperature down to $2 \mathrm{~K}$ and then a rapid drop below $1 \mathrm{~K}$. With increasing magnetic field, the $C_{m} / T$ contribution below $4 \mathrm{~K}$ is steadily suppressed. Unlike the aforementioned quantum spin-liquid magnets with quenched disorder, the zero-field $C_{m} / T$ data lack any upturn at extremely low temperatures [24]. In addition, we could find no scaling and data collapse of $C_{m}[T, H] / T$ in $T / H$ (not shown here).

To understand the failure of the predicted power-law scaling within a bond randomness scenario, we replot $C_{m}$ vs $T$ on a log-log scale in Fig. 3(d). Generally, the bond disorders in a spin liquid and a valence bond create abundant low-energy density of states (DOS) obeying the power-law distribution $N(E) \sim E^{-\alpha}$. A divergent power-law DOS gives rise to the power-law behaviors of $\chi(T) \sim T^{-\alpha}, M(T) \sim$ $H^{1-\alpha}$, and $C(T) \sim T^{1-\alpha}$ (e.g., see Table II in Ref. [25]). In our case, the magnetic specific heat is described by $C_{m}(T) \sim$ $T^{\gamma}$. Obviously, however, the exponent of $\gamma \approx 1.07$ at $\mu_{0} H=$ $0 \mathrm{~T}$ defies the $T^{1-\alpha}$ scaling ( $\left.\alpha=0.26-0.28\right)$ obtained from $M(H)$ and $\chi(T)$. Rather, a nearly $T$-linear contribution is reminiscent of gapless spinon excitations reported in the spinliquid candidate materials [34]. Furthermore, the low- $T$ and high- $H C_{m}$ data display a $T^{2.41}$ dependence, stronger than the $T^{2}$ dependence often reported in the frustrated magnets with the weak bond disorder, obeying the power-law scaling law [24]. Admittedly, there is some uncertainty in evaluating the lattice contribution. As the $\chi^{\prime}[T, H]$ and $M[T, H]$ scalings are limited above $2 \mathrm{~K}$, however, the specific heat anomaly may be resolved by invoking the chemical disorder that introduces partly heterogeneous interactions [see Fig. 1(b)] and, thus, alters extremely low-energy excitations pertaining to $Z_{2}$ fluxes.

To conclude, we determine the ground state and role of quenched disorder in the Kitaev candidate magnet $\mathrm{Cu}_{2} \mathrm{IrO}_{3}$. The ZF- and LF- $\mu$ SR data evidence the formation of a spin-liquid-like ground state, in which the spins remain almost dynamic down to $260 \mathrm{mK}$. This sets up a platform for the bond disordered model on the background of a quantum spin-liquid state, a scenario that is supported by the universal scaling laws we observe in our ac susceptibility and magnetization data. However, the bond randomness scenario seems to be incompatible with the lack of a similar universal scaling in the specific heat data. This highlights a many-sided impact of chemical disorders on a Kitaev honeycomb lattice, which generates not only random exchange interactions but also heterogenous local spin networks [see Fig. 1(b)]. Noticeably, a nearly $T$-linear dependence of $C_{m}$ at $T=$ 1-10 K switches to a much stronger $T$ dependence below 
$1 \mathrm{~K}$, suggesting that the low-energy excitations develop a tiny gap due to the heterogeneous interactions, which is thermally populated by an energy of the order of $1 \mathrm{~K}$.

Recent theoretical calculations show that vison and Majorana zero mode can be created in the vicinity of a site vacancy [35]. These zero-energy resonances are largely hidden to the static spin susceptibility, but they may lead to a breakdown of the low- $T$ specific heat scaling expected from the bond-disordered spin liquid model.

This work was supported by Korea Research Foundation (KRF) Grants (No. 2018-0189 and No. 2018-0099, No. 2013M7A1A1075764) funded by the Korea government (MEST). We acknowledge the support of the HLD at HZDR, a member of the European Magnetic Field Laboratory (EMFL). We acknowledge support from the liquid Helium facility at IISER Mohali.

Note added.-Recently, we noted that a related study by E. M. Kenney et al. was posted in Ref. [36], which observed a coexistence of static and dynamic magnetism. Unlike their $\mu \mathrm{SR}$ data, the absence of frozen magnetic moments is clear in our sample, thereby ruling out a nucleation of the quenched disorder. As to the dynamic magnetism, our data are consistent with their results.

*kchoi@cau.ac.kr

[1] A. Kitaev, Ann. Phys. (Amsterdam) 321, 2 (2006).

[2] G. Baskaran, S. Mandal, and R. Shankar, Phys. Rev. Lett. 98, 247201 (2007).

[3] J. Knolle, D. L. Kovrizhin, J. T. Chalker, and R. Moessner, Phys. Rev. Lett. 112, 207203 (2014).

[4] J. Knolle, G.-W. Chern, D. L. Kovrizhin, R. Moessner, and N. B. Perkins, Phys. Rev. Lett. 113, 187201 (2014).

[5] J. Nasu, M. Udagawa, and Y. Motome, Phys. Rev. Lett. 113, 197205 (2014).

[6] G. Jackeli and G. Khaliullin, Phys. Rev. Lett. 102, 017205 (2009).

[7] J. Chaloupka, G. Jackeli, and G. Khaliullin, Phys. Rev. Lett. 105, 027204 (2010).

[8] J. G. Rau, E.-H. Lee, and H.-Y. Kee, Phys. Rev. Lett. 112, 077204 (2014).

[9] Y. Singh and P. Gegenwart, Phys. Rev. B 82, 064412 (2010).

[10] S. K. Choi, R. Coldea, A. N. Kolmogorov, T. Lancaster, I. I. Mazin, S. J. Blundell, P. G. Radaelli, Y. Singh, P. Gegenwart, K. R. Choi, S.-W. Cheong, P. J. Baker, C. Stock, and J. Taylor, Phys. Rev. Lett. 108, 127204 (2012).

[11] T. Takayama, A. Kato, R. Dinnebier, J. Nuss, H. Kono, L. S. I. Veiga, G. Fabbris, D. Haskel, and H. Takagi, Phys. Rev. Lett. 114, 077202 (2015).

[12] K. A. Modic, T. E. Smidt, I. Kimchi, N.P. Breznay, A. Biffin, S. K. Choi, R. D. Johnson, R. Coldea, P. WatkinsCurry, G. T. McCandless, J. Y. Chan, F. Gandara, Z. Islam, A. Vishwanath, A. Shekhter, R. D. McDonald, and J. G. Analytis, Nat. Commun. 5, 4203 (2014).

[13] A. Glamazda, P. Lemmens, S.-H. Do, Y. S. Choi, and K.-Y. Choi, Nat. Commun. 7, 12286 (2016).
[14] K. W. Plumb, J. P. Clancy, L. J. Sandilands, V. V. Shankar, Y. F. Hu, K. S. Burch, H.-Y. Kee, and Y.-J. Kim, Phys. Rev. B 90, 041112(R) (2014).

[15] A. Banerjee, C. A. Bridges, J.-Q. Yan, A. A. Aczel, L. Li, M. B. Stone, G. E. Granroth, M. D. Lumsden, Y. Yiu, J. Knolle, S. Bhattacharjee, D. L. Kovrizhin, R. Moessner, D. A. Tennant, D. G. Mandrus, and S. E. Nagler, Nat. Mater. 15, 733 (2016).

[16] S.-H. Do, S.-Y. Park, J. Yoshitake, J. Nasu, Y. Motome, Y. S. Kwon, D. T. Adroja, D. J. Voneshen, K. Kim, T.-H. Jang, J.-H. Park, K.-Y. Choi, and S. Ji, Nat. Phys. 13, 1079 (2017).

[17] K. Kitagawa, T. Takayama, Y. Matsumoto, A. Kato, R. Takano, Y. Kishimoto, S. Bette, R. Dinnebier, G. Jackeli, and H. Takagi, Nature (London) 554, 341 (2018).

[18] M. Abramchuk, C. Ozsoy-Keskinbora, J. W. Krizan, K. R. Metz, D. C. Bell, and F. Tafti, J. Am. Chem. Soc. 139, 15371 (2017).

[19] K. Slagle, W. Choi, L. E. Chern, and Y. B. Kim, Phys. Rev. B 97, 115159 (2018).

[20] R. Yadav, R. Ray, M. S. Eldeeb, S. Nishimoto, L. Hozoi, and J. van den Brink, Phys. Rev. Lett. 121, 197203 (2018).

[21] A. Suter and B. M. Wojek, Phys. Procedia 30, 69 (2012).

[22] S.-k. Ma, C. Dasgupta, and C.-k. Hu, Phys. Rev. Lett. 43, 1434 (1979).

[23] J. E. Hirsch and J. V. Jose, Phys. Rev. B 22, 5339 (1980).

[24] I. Kimchi, J. P. Sheckelton, T. M. McQueen, and P. A. Lee, Nat. Commun. 9, 4367 (2018).

[25] I. Kimchi, A. Nahum, and T. Senthil, Phys. Rev. X 8, 031028 (2018).

[26] J. Knolle, R. Moessner, and N. B. Perkins, Phys. Rev. Lett. 122, 047202 (2019).

[27] A. Yaouanc and P. Dalmas de Rotier, Muon Spin Rotation, Relaxation, and Resonance: Applications to Condensed Matter (Oxford University Press, Oxford, 2011).

[28] J. S. Gardner, S. R. Dunsiger, B. D. Gaulin, M. J. P. Gingras, J. E. Greedan, R. F. Kiefl, M. D. Lumsden, W. A. MacFarlane, N. P. Raju, J. E. Sonier, I. Swainson, and Z. Tun, Phys. Rev. Lett. 82, 1012 (1999).

[29] P. Mendels, F. Bert, M. A. de Vries, A. Olariu, A. Harrison, F. Duc, J. C. Trombe, J. S. Lord, A. Amato, and C. Baines, Phys. Rev. Lett. 98, 077204 (2007).

[30] Y. Li, D. Adroja, P. K. Biswas, P. J. Baker, Q. Zhang, J. Liu, A. A. Tsirlin, P. Gegenwart, and Q. Zhang, Phys. Rev. Lett. 117, 097201 (2016).

[31] A. Yaouanc, P. Dalmas de Reotier, A. Bertin, C. Marin, E. Lhotel, A. Amato, and C. Baines, Phys. Rev. B 91, 104427 (2015).

[32] I. A. Campbell, A. Amato, F. N. Gygax, D. Herlach, A. Schenck, R. Cywinski, and S. H. Kilcoyne, Phys. Rev. Lett. 72, 1291 (1994).

[33] A. G. Redfield, IBM J. Res. Dev. 1, 19 (1957).

[34] W.-J. Lee, S.-H. Do, S. Yoon, S. Lee, Y. S. Choi, D. J. Jang, M. Brando, M. Lee, E. S. Choi, S. Ji, Z. H. Jang, B. J. Suh, and K.-Y. Choi, Phys. Rev. B 96, 014432 (2017).

[35] M. Udagawa, Phys. Rev. B 98, 220404 (2018).

[36] E. M. Kenney, C. U. Segre, W. Lafargue-Dit-Hauret, O. I. Lebedev, M. Abramchuk, A. Berlie, S. P. Cottrell, G. Simutis, F. Bahrami, N. E. Mordvinova, J. L. McChesney, G. Fabbris, D. Haskel, X. Rocquefelte, M. J. Graf, and F. Tafti, arXiv:1811.00565. 